\title{
The major histocompatibility complex in Old World camelids and low polymorphism of its class II genes
}

\author{
Martin Plasil ${ }^{1,2}$, Elmira Mohandesan ${ }^{3,4}$, Robert R. Fitak ${ }^{4,5}$, Petra Musilova ${ }^{6}$, Svatava Kubickova ${ }^{6}$, \\ Pamela A. Burger ${ }^{3}$ and Petr Horin ${ }^{1,2^{*}}$
}

\begin{abstract}
Background: The Major Histocompatibility Complex (MHC) is a genomic region containing genes with crucial roles in immune responses. MHC class I and class II genes encode antigen-presenting molecules expressed on the cell surface. To counteract the high variability of pathogens, the $\mathrm{MHC}$ evolved into a region of considerable heterogeneity in its organization, number and extent of polymorphism. Studies of MHCs in different model species contribute to our understanding of mechanisms of immunity, diseases and their evolution. Camels are economically important domestic animals and interesting biomodels. Three species of Old World camels have been recognized: the dromedary (Camelus dromedarius), Bactrian camel (Camelus bactrianus) and the wild camel (Camelus ferus). Despite their importance, little is known about the MHC genomic region, its organization and diversity in camels. The objectives of this study were to identify, map and characterize the MHC region of Old World camelids, with special attention to genetic variation at selected class MHC II loci.
\end{abstract}

Results: Physical mapping located the MHC region to the chromosome 20 in Camelus dromedarius. Cytogenetic and comparative analyses of whole genome sequences showed that the order of the three major sub-regions is "Centromere - Class II - Class III - Class I". DRA, DRB, DQA and DQB exon 2 sequences encoding the antigen binding site of the corresponding class II antigen presenting molecules showed high degree of sequence similarity and extensive allele sharing across the three species. Unexpectedly low extent of polymorphism with low numbers of alleles and haplotypes was observed in all species, despite different geographic origins of the camels analyzed. The DRA locus was found to be polymorphic, with three alleles shared by all three species. DRA and DQA sequences retrieved from ancient DNA samples of Camelus dromedarius suggested that additional polymorphism might exist.

Conclusions: This study provided evidence that camels possess an MHC comparable to other mammalian species in terms of its genomic localization, organization and sequence similarity. We described ancient variation at the DRA locus, monomorphic in most species. The extent of molecular diversity of MHC class II genes seems to be substantially lower in Old World camels than in other mammalian species.

Keywords: MHC, Exon 2, Wild two-humped camel, Bactrian camel, Dromedary, Camelini, aDNA

\section{Background}

Pathogens are considered to be one of the driving forces of evolution. The major histocompatibility complex (MHC) is a genomic region containing immune response (IR) genes, which play a crucial role in host and pathogen interactions. MHC class I and class II genes encode antigen-presenting

\footnotetext{
* Correspondence: horin@dior.ics.muni.cz

'Department of Animal Genetics, Veterinary and Pharmaceutical University, Brno, Czech Republic

${ }^{2}$ Ceitec VFU, RG Animal Immunogenomics, Brno, Czech Republic

Full list of author information is available at the end of the article
}

molecules responsible for the dual recognition of antigenic peptides on the cell surface [1]. MHC-encoded antigen presenting molecules are thus directly involved in molecular interactions with specific peptides derived from pathogens to which a population is exposed. Therefore, the MHC genes are under strong selective pressure and contain signatures of both positive and negative selection [2]. To counteract the high variability of pathogens and pathogen-derived molecules, the MHC of Gnathostomata evolved into a region of considerable heterogeneity in its 
organization, number, and extent of polymorphism both within and between species [3-5]. Spanning approximately 4 megabases $(\mathrm{Mb})$, the $\mathrm{MHC}$ region consists of hundreds of different genes with a variety of functions including antigen presentation and processing as well as non-immune processes [1]. Consequently, MHC class I and class II genes are amongst the most polymorphic genes studied in vertebrates with more than 100 alleles reported in different species, including humans [6, 7]. In MHC class II genes, a majority of the functionally important polymorphisms are concentrated in exon 2, which encodes the antigen-binding site of the molecule $[8,9]$. This diversity is correlated with pathogen richness [10].

Infectious diseases of livestock have a significant economical impact on animal husbandry, and they also may affect human health directly or through food chains. Studies of MHCs in different model species contribute to our understanding of mechanisms of infectious diseases. Economically as well as culturally important among domestic animals are camels, with their long history of adaptation to arid environments and with their capability of providing transport and various commodities important for human development [11-13]. Currently, three extant species of Old World camels are recognized [14-16]. The dromedary (Camelus dromedarius) and Bactrian camel (Camelus bactrianus) are key domestic species in semi-arid and desert areas and are used for food production and camel racing throughout many Arabian, Northern African and Asian countries. The only surviving and critically endangered wild camel species (Camelus ferus) is closely related to them and diverged from the domestic Bactrian camel approximately 1,000,000 years ago [14, 15].

In terms of infectious disease, the Old World camels (genus Camelus) are an interesting biomodel. They are resistant to serious infections threatening other livestock inhabiting the same geographical regions $[17,18]$. Recently, dromedaries have been identified as potential vectors of the Middle East Respiratory Syndrome (MERS) virus [19, 20]. The immune system of camels displays characteristic features of practical importance, like heavy chain antibody homodimers [21, 22]. Furthermore, all extant Camelus species are renowned for their ability to cope with harsh environmental challenges, including high temperatures, drought, and famine combined with high level of physical activities. However, little is known about the MHC genomic region, its organization and diversity in camels [23].

Recently, draft genome sequences have been made available for all three species [13, 16, 24, 25]. Although some MHC genes have been annotated in these assemblies, the draft genome sequences still contain gaps and errors [25]. It has been repeatedly recognized for other species, that the complexity of the MHC and other complex regions involved in mechanisms of immunity and disease cannot be resolved at this level [26]. Moreover, in camels the full genome sequences available were derived from single individuals, while the complexity of MHC and of its sub-regions should be based on targeted re-sequencing of multiple individuals originating from genetically different populations [27].

Therefore, the objectives of this study were to i) identify and map the MHC region in the genomes of Old World camelids, ii) characterize its overall genomic organization, and iii) characterize the genetic variation at selected class MHC II loci in modern and ancient samples.

\section{Methods}

\section{Sample collection and DNA extraction}

Peripheral blood from different populations of Mongolian Bactrian camels $(C$. bactrianus, $n=57)$ and dromedaries from Jordan $(C$. dromedarius, $n=31)$ was collected during routine veterinary procedures. DNA extractions were performed using the NucleoSpin ${ }^{\circ}$ Blood kit (MachereyNagel) or the standard phenol-chloroform extraction [28]. Additionally, we acquired previously extracted DNA from different geographic populations of $C$. dromedarius $(n=35), C$. bactrianus $(n=1)$, and C. ferus $(n=20)$ from the sample database stored at the Vetmeduni Vienna, Research Institute of Wildlife Ecology (Additional file 1). These samples represent a majority of the geographical range within each camel species, thus maximizing our ability to sample the breadth of genetic diversity. All DNA samples were stored at $-20{ }^{\circ} \mathrm{C}$ prior to analysis. The numbers of individuals from each species, which were analyzed for the MHC class II genes, are presented in Table 1.

In addition to the modern samples collected above, ancient dromedary specimens $(n=3)$ were collected from an archaeological site in Jordan (Aqaba) as part of a separate study (Mohandesan et al. submitted). The samples stem from the Mamluk and Ottoman period, ranging from 250 to 740 years ago (Table 2). The ancient dromedary specimens were prepared in a dedicated ancient DNA laboratory at the Paleogenetic Core Facility of the ArchaeoBioCenter at the Ludwig-Maximilian-University, Munich, Germany, following a range of standard contamination precautions [29]. DNA was extracted from bone material following the protocols described in Rohland and Hofreiter [30], and Rohland et al. [31]. Extractions were conducted in batches of seven samples and one extraction blank as a control.

\section{Physical mapping of the MHC region}

Fluorescence in situ hybridization (FISH) probes were designed to physically map the MHC region and to establish

Table 1 Total numbers of Old World camelids analyzed for MHC class II genes

\begin{tabular}{lllll}
\hline Species & DRA $(n)$ & DRB $(n)$ & DQA $(n)$ & DQB $(n)$ \\
\hline Camelus ferus & 18 & 11 & 8 & 5 \\
Camelus bactrianus & 30 & 42 & 47 & 18 \\
Camelus dromedarius & 37 & 54 & 19 & 7 \\
\hline
\end{tabular}


Table 2 Historical dromedary camel samples used in this study

\begin{tabular}{lllll}
\hline Sample ID & Site & Excavation year & Period (Dates) & $\begin{array}{l}\text { Gene partially } \\
\text { recovered }\end{array}$ \\
\hline AQ30 & Jordan & 2006 & Mamluk (1260-1456 AD) & DRA \\
AQ34 & Jordan & 2006 & Mamluk (1260-1456 AD) & DRA \\
AQ40 & Jordan & 2007 & Ottoman (1456-1870 AD) & DQA \\
\hline
\end{tabular}

its orientation relative to the centromere. One FISH probe specific for the class I region (MHCI) was placed on gene TRIM39 at the scaffold KN277189.1 (positions: 9966611006833, and a class II specific probe (MHCII) was placed on gene FANCA at the scaffold KN276514.1 (positions: 2132659-2136283). Both scaffolds are part of the Bactrian camel genome assembly [GenBank: JARL00000000.1]. The primers used for amplifying the FISH probes are listed in Table 3. The PCR products were cloned into the pDrive Cloning Vector (Qiagen) and the recombinant plasmids were labeled with digoxigenin-11-dUTP or biotin-16-dUTP (Roche Diagnostics GmbH, Mannheim, Germany) using the Nick Translation Reagent Kit (Vysis, Richmond, UK). The labeled probes were used for standard FISH to dromedary metaphase chromosomes prepared from peripheral blood culture [32]. Hybridization of MHCI and MHCII probes were visualized by immunodetection using fluorescein avidin (Vector Laboratories, Burlingame, CA, USA) or anti-digoxigenin-rhodamine (Roche), respectively.

\section{Overall genomic organization of the MHC region in Old World camelids}

One of the goals in this study was to assess the order of the three major MHC sub-regions, i.e. class I, II and III. Recently sequenced genomes of domestic Bactrian and dromedary camels $[13,25]$ were analyzed to decipher the overall organization of MHC region in camels. For this purpose, class-specific but adjacent sequences located at the boundaries between the class I, II and III regions and likely to be located within the same contigs were identified in the assembled reference bovine genome Btau3.5 (Table 4). A standard BLAST search [33] of all camelid genomic resources available was then performed by using these sequences to assess their physical proximity in the (fragmented) camel genomes.

\section{Amplification and sequencing of MHC class II genes from modern camels}

Due to their functional importance, we specifically focused on the analysis of the exon 2 coding sequences of four genes, namely $D R A, D R B, D Q A$ and $D Q B$. Camel-specific primers were designed using the Primer3 software [34]. For this purpose, species- and locus-specific regions were identified by BLAST [33] search of bovine $D R A, D R B$, $D Q A$, and $D Q B$, exon 2 sequences against the wild
Bactrian camel draft genome assembly [16]. This approach was successful for all loci except $D Q B$, because no $D Q B$ like sequences were found in the draft genomes available. In a second step, based on the camel-specific sequences retrieved during the first round of amplifications, primers located in the neighboring introns and amplifying the fulllength exon 2 sequences could be designed. In addition, we developed a set of primers specific for each locus separately to check possible allelic dropouts (Table 3). As for $D Q B$, attempts to use bovine primers DQB-LA40, DQB-LA41 and DQB-LA48 [35] failed. Eventually, the zoo-primers GH28 and GH29 amplifying $D Q B$ exon 2 in various mammalian species were used successfully [36]. All primer sequences and resulting PCR product lengths are summarized in Table 3.

The PCR reactions were performed in a reaction volume of $12.5 \mu \mathrm{l}$ containing $50 \mu \mathrm{g} / \mathrm{ml}$ of DNA, 1x KAPA2G Buffer A (with $\mathrm{MgCl}_{2}$ ), 1x KAPA Enhancer 1, $0.2 \mathrm{mM}$ of each dNTPs, $0.5 \mu \mathrm{M}$ of forward and reverse primer and $0.5 \mathrm{U}$ of KAPA2G Robust HotStart DNA Polymerase (Kapa Biosystems, USA). Negative controls were included in each PCR. Amplified PCR products were purified with ExoSAP-IT using standard protocol (Affymetrix, USA) and subjected to Sanger sequencing (Macrogen Europe, the Netherlands). Next generation sequencing (NGS) was used for a subset of samples $(n=20)$ as a part of our task to have all variants confirmed based on at least two independent PCRs. For the remaining samples, Sanger data available allowed the confirmation. Two platforms, Roche GS Junior or Illumina MiSeq were used according to standard protocols.

Data from Roche GS Junior were converted from sff to fasta format using sff_extract 0.3.0 [37]. Individual libraries within.sff file were isolated using sfffile command included in the Roche Data Analysis V2.9 [38]. Data from GS Junior and MiSeq were checked using FastQC quality control tool [39]. All reads were trimmed with QTrim v1.1 [40] and quality filtered with $-\mathrm{q} 20$ using cutadapt_v1.4.1 [41]. Trimmed reads were aligned to the wild camel genome reference sequence [16] [Genbank: AGVR00000000] using BWA v0.6.2 [42] with default parameters. We identified polymorphisms using Samtools v1.2 [43] and viewed results in IGViewer 2.3 [44]. For further analysis of polymorphisms, the GATK UnifiedGenotyper v2.7-2 [45] was used with the following settings [46]: sample ploidy $=40$, site quality prior $=20$, standard min confidence threshold for calling $=30$, minimum power threshold for calling $=0.95$. 
Table 3 Primers used to amplify different MHC sequences in Old World camelids

\begin{tabular}{|c|c|c|c|c|}
\hline Locus & Name & Sequence $\left(5^{\prime} \rightarrow 3^{\prime}\right)$ & Purpose & PCR product length \\
\hline \multirow[t]{10}{*}{ DRA } & DRA-EXON2-F & TGAGAATITTGGTTTGCTTATGGCA & Bact, drom & $514 \mathrm{bp}$ \\
\hline & DRA-EXON2-R & CCTCTGAGCAACACGAACGTCCTTCA & & \\
\hline & DRA-EXON2-WC-F & TGGGTGTTTCAGCTCTTGTG & Ferus & $650 \mathrm{bp}$ \\
\hline & DRA-EXON2-WC-R & AGATACCATGGGTGGCAAAG & & \\
\hline & DRA-EXON2-3-F & CCCTGGAATTCGGGTTTAAG & Preventing allele dropout & $923 \mathrm{bp}$ \\
\hline & DRA-EXON2-3-R & GGCTGAAAAAGCAGTTGAGC & & \\
\hline & DRA_F1 & TGATCATCCAGGCTGAGTTC & Ancient samples & $139 \mathrm{bp}$ \\
\hline & DRA_R1 & GCAAACCGTCCAAATTCTTC & & \\
\hline & DRA_F2 & CGTGGATCTGGAAAAGAAGG & Ancient samples & $154 \mathrm{bp}$ \\
\hline & DRA_R2 & ATTGGTGTTCGGGGTGTG & & \\
\hline \multirow[t]{6}{*}{ DRB } & DRB-EXON2-2-F & AGCAGTGGGGGTCCTAGTG & Bact, drom & $457 \mathrm{bp}$ \\
\hline & DRB-EXON2-2-R & ACCCACCCGGACTCAGTATC & & \\
\hline & DRB-EXON2-WC-F & TTCAGGAGGAGGTGGTGATG & Ferus & 764 bp \\
\hline & DRB-EXON2-WC-R & CTCAGACCCCAGACCCATT & & \\
\hline & DRB-EXON2-3-F & GCCAGCCCTAGGCAAGTAAG & Preventing allele dropout & 852 bp \\
\hline & DRB-EXON2-3-R & GTTCTCTCAGACCCCAGACC & & \\
\hline \multirow[t]{12}{*}{ DQA } & DQA1-EXON2-F & CATGAAAGTCAATTTATCCTGTCAC & Bact & $351 \mathrm{bp}$ \\
\hline & DQA-EXON2-2-R & AGTGAGGCCTGGTATGAAGG & & \\
\hline & DQA-EXON2-WC-F & ACGTGCTGGGAATTTTGTCT & Drom & $414 \mathrm{bp}$ \\
\hline & DQA-EXON2-2-R & AGTGAGGCCTGGTATGAAGG & & \\
\hline & DQA-EXON2-WC-F & ACGTGCTGGGAATTTTGTCT & Ferus & $646 \mathrm{bp}$ \\
\hline & DQA-EXON2-WC-R & GCTATGGGAGCTTTCCTTGA & & \\
\hline & DQA-EXON2-4-F & ATGGTGCAGAGAGCAGAAGG & Preventing allele dropout & 1096 bp \\
\hline & DQA-EXON2-4-R & TGGGAAACACAGTCACCTCA & & \\
\hline & DQA_F1 & YGGCRTAAATGTCTACCAGTC & Ancient samples & 133 bp \\
\hline & DQA_R1 & TSRAAACTTGSTAAACAGAGG & & \\
\hline & DQA_F2 & CGTGGACTTGGAGAAGAAGG & Ancient samples & $145 \mathrm{bp}$ \\
\hline & DQA_R2 & AGCAGTAGAGTTGGAGCGTTT & & \\
\hline \multirow[t]{2}{*}{ DQB } & GH 28 & CTCGGATCCGCATGTGCTACTTCACCAACG & Bact, drom, ferus & $181 \mathrm{bp}$ \\
\hline & GH 29 & GAGCTGCAGGTAGTTGTGTCTGCACAC & & \\
\hline \multirow[t]{2}{*}{ FANCA } & KN276514-F & TCAAGTCAGAAACAAGAACACAGA & FISH probe MHCII & $3574 \mathrm{bp}$ \\
\hline & KN276514-R & CATCATTGCCAGATCCTTCA & & \\
\hline \multirow[t]{2}{*}{ TRIM39 } & KN277189-F & CCACCCACCATGTGTATGAA & FISH probe $\mathrm{MHCl}$ & 3459 bp \\
\hline & KN277189-R & GCTGAGAGTGCAGGGATAGG & & \\
\hline
\end{tabular}

Bact - Primer pair used to amplify samples of Camelus bactrianus. Drom - Primer pair used to amplify samples of Camelus dromedarius. Ferus - Primer pair used to amplify samples of Camelus ferus

Table 4 Locations of BLAST hits on the Bactrian genome scaffolds KN276514.1 and KN277189.1 (Accession number JARL00000000.1)

\begin{tabular}{|c|c|c|c|c|c|}
\hline Gene (cattle) & Scaffold & Start & End & ID & Boundaries \\
\hline DQB exon 2 & KN276514.1 & 4092056 & 4091824 & S43263.1|53-319 & MHC class II \\
\hline butyrophilin-like protein 1-like & & 4159484 & 4168470 & LOC504295 & MHC class II scaffold \\
\hline butyrophilin subfamily 1 member A1 & KN277189.1 & 226 & 1044 & LOC615223 & MHC class I \& III scaffold \\
\hline $\mathrm{NOTCH} 4$ & & 17978 & 41342 & 536128 & MHC class III \\
\hline BAT1 & & 513067 & 522625 & 540191 & MHC class III \\
\hline BoLA, MHC class I A & & 1067171 & 1157210 & 533050 & MHC class I \\
\hline
\end{tabular}


The polymorphisms identified from the NGS data were validated by cloning and Sanger sequencing. Amplicons from selected heterozygous individuals were ligated to the pJET1.2/blunt vector and transformed using the CloneJet ${ }^{\text {Ts }}$ PCR cloning kit (Fermentas) according to the manufacturer's instructions. Escherichia coli TOP 10 were used as competent cells. Colonies were screened for inserts of the expected length ( 200 - $800 \mathrm{bp})$ by PCR, using the amplicon-specific primers. Purified PCR products were Sanger sequenced (Macrogen Europe). BioEdit 7.1.3.0 with implemented ClustalW algorithm for multiple alignments was used for the quality-check of the generated data using the ABI 3730XL DNA Analyzer [47].

\section{Amplification and sequencing of MHC class II genes from ancient dromedary samples}

Exons 2 of two MHC genes, DRA (246 bp) and $D Q A$ (249 bp) from the ancient dromedary specimens were analyzed. Two overlapping regions (fragments of $138 \mathrm{bp}$ and $237 \mathrm{bp}$ ) of each gene were amplified and sequenced (Table 2). The PCR amplification was carried out in $20 \mu \mathrm{l}$ volume containing 1x PCR buffer (Invitrogen), $4 \mathrm{mM} \mathrm{MgCl}_{2}$ (Invitrogen), $1 \mathrm{mg} / \mathrm{ml} \mathrm{BSA} \mathrm{(Invitrogen),}$ $250 \mu \mathrm{M}$ mix dNTPs (Invitrogen), $1.5 \mu \mathrm{M}$ for each primer (Invitrogen), $0.5 \mathrm{U}$ of AmpliTaq Gold (Invitrogen) and $5 \mu \mathrm{l}$ DNA template. The PCR reactions were amplified using an iCycler ${ }^{\text {тм }}$ Thermal cycler (Bio-RAD) located in a separate facility. The amplification programme consisted of initial denaturation at $94{ }^{\circ} \mathrm{C}$ for $9 \mathrm{~min}$ followed by 60 cycles $\left(94{ }^{\circ} \mathrm{C}\right.$ for $20 \mathrm{~s}, 55^{\circ} \mathrm{C}$ for $30 \mathrm{~s}, 72{ }^{\circ} \mathrm{C}$ for $30 \mathrm{~s}$ ) and a final extension of $72{ }^{\circ} \mathrm{C}$ for $4 \mathrm{~min}$. The PCR products were purified using the QIAquick PCR purification kit (Qiagen) and sequenced in both directions on ABI 3730 XL-Analyzer (Eurofins MWG GmbH, Ebersberg, Germany). Each sequence position was determined from two independent PCR amplifications in both directions to avoid sequence errors caused by template damage. The MHC sequences obtained from the ancient samples were aligned to the MHC DQA exon 2 [GenBank: AGVR01020882.1|:14962-15210] and DRA exon 2 [GenBank: AGVR01020883.1|:30918-31163] reference sequences using CodonCode Aligner v.3.7.1.2 (Codon Code Corporation, USA) and compared to the NCBI nucleotide database sequences, using the BLAST [33] with default blastn parameters.

\section{Cross-validation of MHC class II alleles with genome re- sequencing data of dromedaries}

To further validate the dromedary alleles for the MHC class II genes $D R A, D R B, D Q A$ and $D Q B$, all Sanger sequences retrieved were compared to whole genome sequence (WGS) data obtained from a genome re-sequencing project of nine dromedaries (Fitak et al. in prep). Briefly, all sequencing was performed using an Illumina HiSeq and reads were trimmed and aligned to the dromedary reference [GenBank: GCA_000803125.1] [25]. We realigned reads near insertiondeletion polymorphisms, recalibrated the base quality scores, and identified variants according to the recommended guidelines for the Genome Analysis Toolkit v3.1-1 [46, 48]. We identified the genomic location of the MHC class II genes in the dromedary reference using a nucleotide BLAST [33] search. We compared these regions with the variants identified above and inferred haplotypes when phase could be unambiguously determined $(\leq 1$ heterozygous genotype in the locus). Frequency of heterozygotes was calculated based on Sanger sequences as a proportion of heterozygotes out of all individual camels sequenced.

\section{Phylogenetic analysis}

BioEdit 7.1.3.0 with ClustalW algorithm was used to align the allelic sequences obtained [47]. The Jukes-Cantor [49] and Kimura-2-parameter [50] were chosen as best-fit evolutionary models based on the Akaike Information Criterion with correction for small sample size [51] to build distance matrices and to construct neighbor-joining phylogenetic trees [52] using MEGA6 [53]. Vicugna pacos DRA exon 2 [GenBank: ABRR02040968.1|:530-775], DRB exon 2 [GenBank: ABRR02040966.1|:2004-2273] and DQA exon 2 [GenBank: ABRR02040956.1|:13508-13756] sequences were used as outgroups. We assessed the nodal support in the phylogenetic tree using 1000 bootstrap replications.

General recommendations by Klein et al. [54] were followed for the designation of the camel MHC loci, Mhc Cafe (C. ferus), Mhc Caba (C. bactrianus) and Mhc Cadr (C. dromedarius), respectively. Allele numbers followed the region and locus abbreviation (e.g. MhcCafe-DRA*01). For alleles that could not be assigned to a specific locus, twodigit numbers after the asterisk symbol (*) were used. The accession numbers for alleles are GenBank: KT936396KT936421.

\section{Results \\ Physical mapping of the MHC region}

The two FISH probes targeting the two MHC regions mapped to dromedary chromosome 20q12 according to the dromedary idiogram published by Balmus et al. [55]. It also showed that the class II region is closer to the centromere and the class I region more distant (Fig. 1).

\section{Overall organization of the $\mathrm{MHC}$ region in domestic Bactrian camels}

The class II region containing the $D R A, D R B, D Q A$, and $D Q B$ genes was found to be located at the end of the Bactrian genome scaffold KN276514.1 between positions 3993442 and 4091824 (see Table 4). The end of this scaffold contains the butyrophilin like subfamily 1 member A1 associated with the MHC class II in other 




Fig. $1 \mathrm{FISH}$ MHC probes on dromedary chromosomes. Partial metaphase spread after hybridization of the probe $\mathrm{MHCl}$ (red) and $\mathrm{MHCll}$ (green) of dromedary sample. Chromosomes were counterstained with DAPI

mammalian species [56]. Another butyrophilin-like gene was found on scaffold KN277189.1 (GenBank), physically linked to the MHC class III region genes NOTCH4 and BAT1. At the end of this scaffold, the MHC class I locus A sequence was identified. These data provide information on the adjacency of both MHC class I and II regions with MHC class III loci. Based on these sequence analyses, the overall genomic organization of the Bactrian camel MHC is suggested as displayed in Fig. 2.

\section{Exon 2 polymorphisms of MHC class II genes in Old World camelids}

DRA exon 2. The exon 2 of the DRA gene spans 246 bp in all three species. The exon contains two single nucleotide polymorphisms (SNPs), one synonymous and one non-synonymous, shared by the three species (Fig. 3). The combination of the two SNPs produced three different alleles shared again by all three species (Fig. 3). The frequencies of heterozygotes were 0.61 for wild camels, 0.53 for Bactrian camels and 0.32 for dromedaries. These differences were not statistically significant at $p=0.08$ (chi-square 5.05). The distribution of the alleles in the groups analyzed is shown in Table 5. The corresponding phylogenetic tree is displayed in Fig. 4a. Selected chromatograms are available in Additional file 2.

We successfully amplified and sequenced two partial $D R A$ exon 2 sequences from three ancient dromedaries

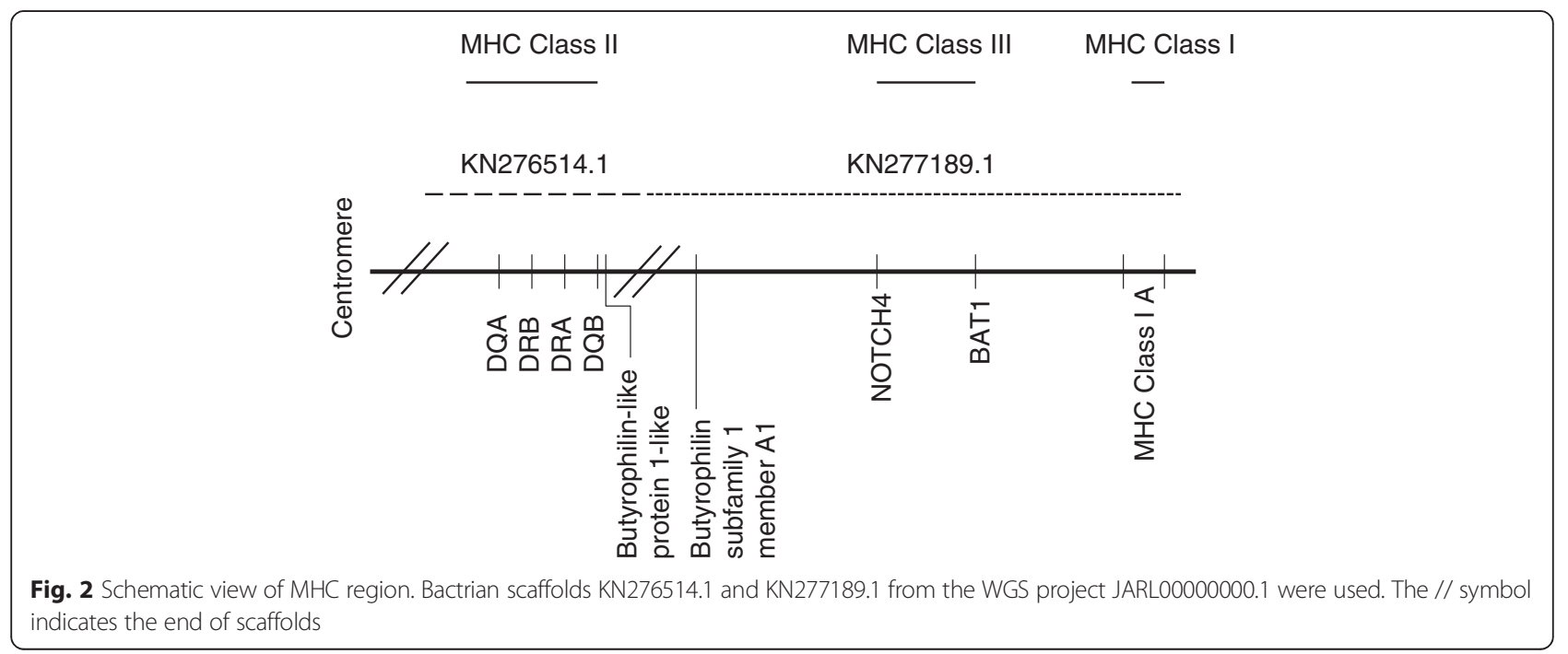




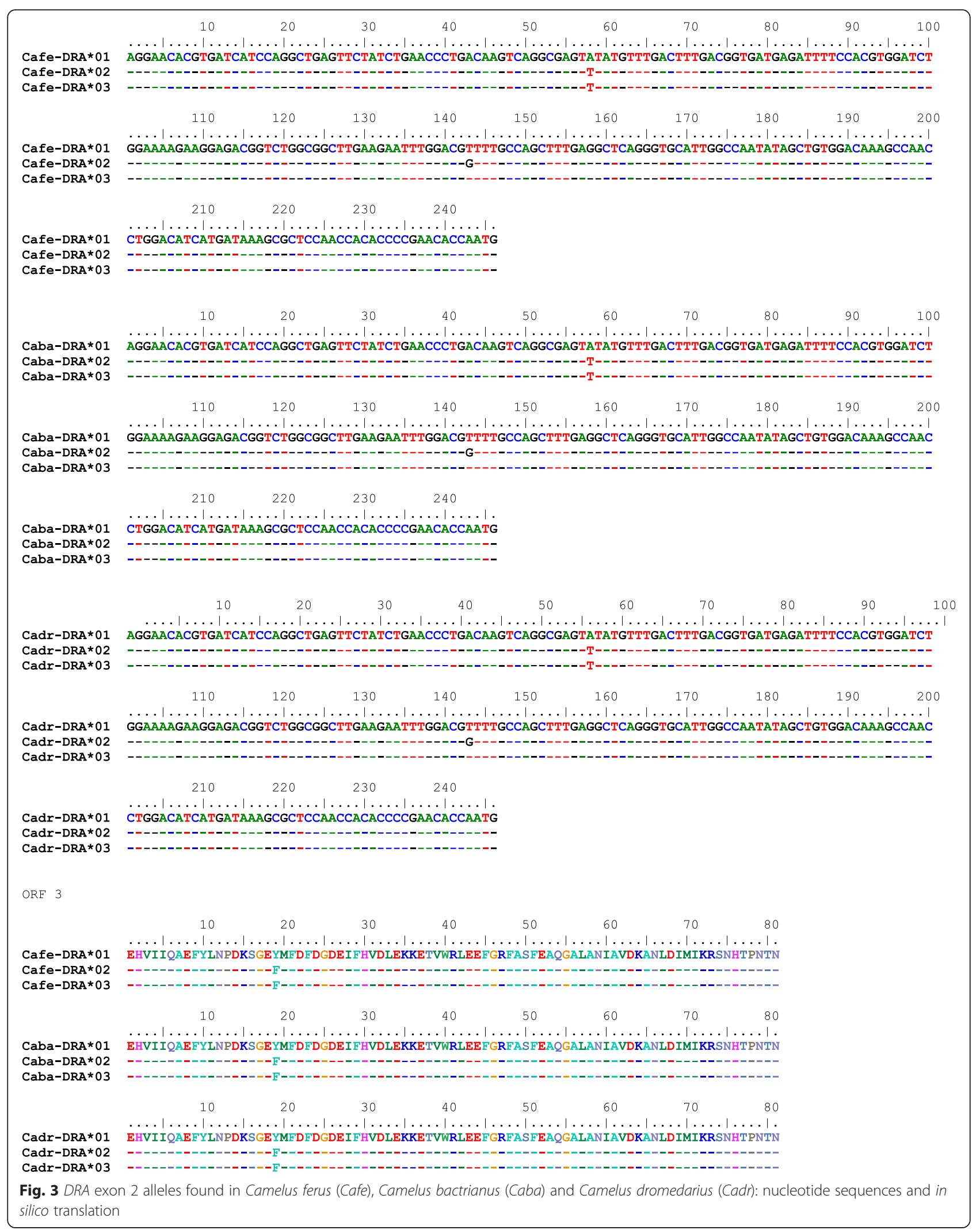


Table 5 Distribution of MHC class II exon 2 allelic sequences in the three camel species observed in Sanger sequenced samples

\begin{tabular}{|c|c|c|c|c|c|c|c|c|c|c|c|c|c|c|}
\hline & & DRA a & & & & DRB all & & & & & & $\mathrm{DQA} a$ & & \\
\hline & (n) & ${ }^{*} 01$ & ${ }^{*} 02$ & ${ }^{*} 03$ & (n) & ${ }^{*} 01$ & ${ }^{*} 02$ & ${ }^{*} 03$ & ${ }^{*} 04$ & ${ }^{*} 05$ & (n) & ${ }^{*} 01$ & ${ }^{*} 02$ & ${ }^{*} 03$ \\
\hline Cafe & 18 & 0.306 & 0.25 & 0.444 & 11 & 0.409 & 0.591 & - & - & & 8 & 0.875 & 0.125 & - \\
\hline Caba & 30 & 0.433 & 0.517 & 0.05 & 42 & 0.702 & 0.048 & 0.048 & 0.202 & & 47 & 0.17 & 0.809 & 0.021 \\
\hline Cadr & 37 & 0.183 & 0.75 & 0.067 & 54 & 0.296 & 0.463 & 0.13 & 0.102 & 0.009 & 19 & 1 & - & - \\
\hline
\end{tabular}



Fig. 4 Evolutionary trees of a DRA exon 2 (sum of branch length $=0.12597149$ ), $\mathbf{b}$ DRB exon 2 (sum of branch length $=0.28248445$ ) and $\mathbf{c} D Q A$ exon 2 in camelids (sum of branch length $=0.25550596$ ). Bootstrap support is shown for each node as a percentage based upon 1000 replicates 
(AQ30, AQ34; Table 2). The sequence recovered from AQ34 was identical to $C a d r-D R A * 02$, while AQ30 showed an additional three $\mathrm{C} / \mathrm{T}$ substitutions when compared with the reference sequence (Fig. 5).
$D R B$ exon 2

The $D R B$ exon 2 spans $270 \mathrm{bp}$ and contains five polymorphic sites across the three species. While $C$. ferus harbors two synonymous variants (Fig. 6), two additional

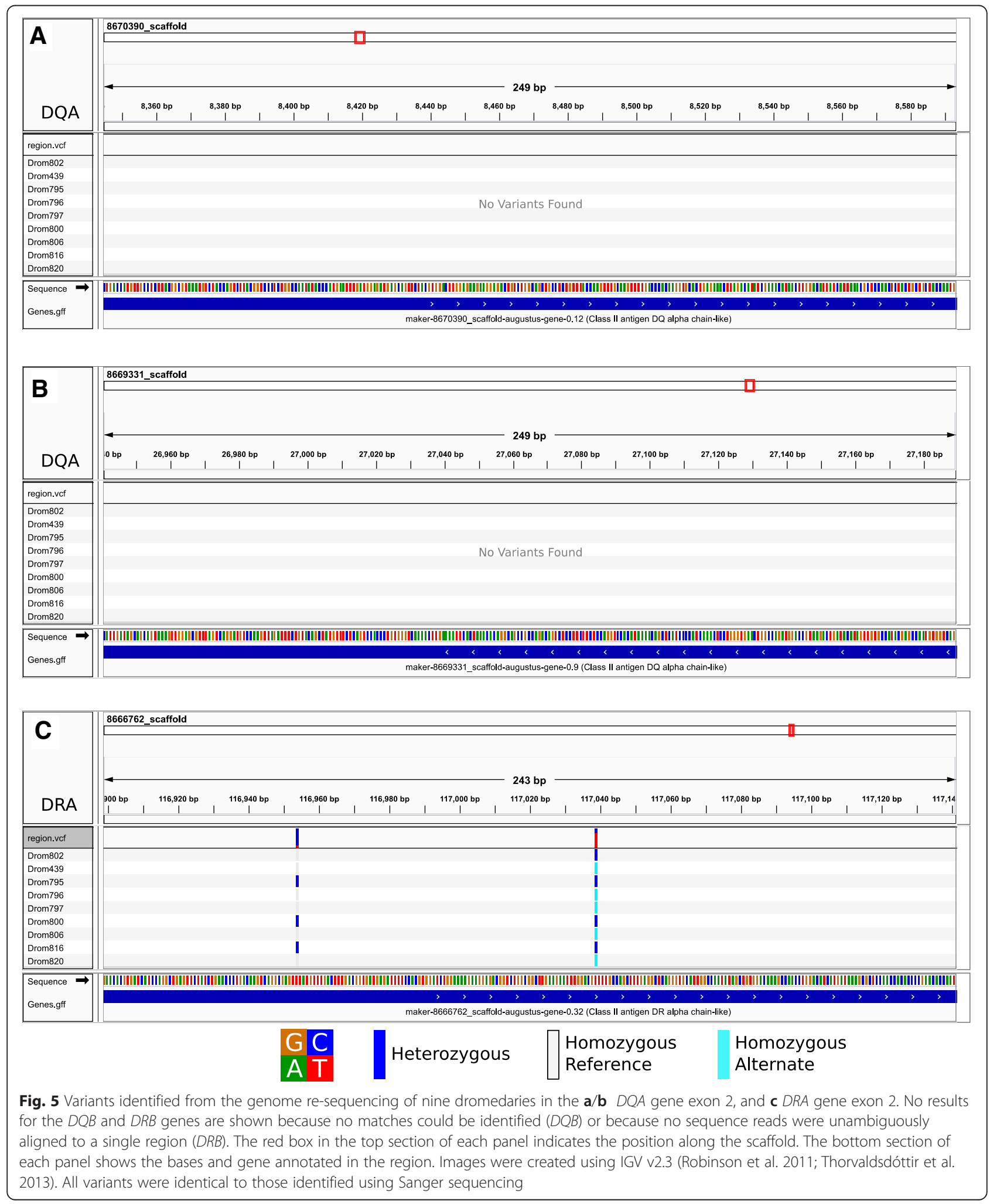






variants were found in $C$. bactrianus. A single nonsynonymous substitution (arginine to tryptophan) was found at nucleotide position 264. Combined with C. ferus, four alleles were identified (Fig. 6). The distribution of the alleles among species is shown in Table 5. The frequencies of heterozygotes were 0.27 for wild camels, 0.48 for Bactrian camels and 0.59 for dromedaries. The differences were not statistically significant ( $p=0.13$; chi-square 4.14). The respective phylogenetic tree is presented in Fig. $4 \mathrm{~b}$. Due to reads that could not be unambiguously aligned to a single region (two matching copies of $D R B$ were found, Table 6), SNPs in the $D R B$ exon 2 could not be validated like in the other exons analyzed. Selected chromatograms are available in Additional file 3.

\section{$D Q A$ exon 2}

Eleven polymorphic sites were observed in the $D Q A$ exon 2 of $C$. bactrianus, nine of them also in C. ferus. Four SNPs were synonymous substitutions (Fig. 7). Three haplotypes (alleles) were identified (Fig. 7), one of them shared among the three species and another one shared between wild and Bactrian camels. The distribution of alleles among species is shown in Table 5. The frequencies of heterozygotes were 0.25 for wild camels, 0.09 for Bactrian camels and 0.00 for dromedaries. The differences were not statistically significant $(p=0.09$; chi-square 4.75). The phylogenetic tree is shown in Fig. 4c. By using in silico analysis of the dromedary NGS data, no variation was identified in $D Q A$ exon 2 , which

Table 6 Location of MHC Class II genes in the dromedary reference genome (Accession GCA_000803125.1) and the number of SNPS identified from Sanger $\left(S_{\text {SANGER }}\right)$ and next-generation $\left(S_{N G S}\right)$ sequencing

\begin{tabular}{|c|c|c|c|c|c|c|}
\hline Gene & Scaffold & Start & End & Match \% & S SANGER $_{2}$ & $S_{\text {NGS }}$ \\
\hline DRA Exon 2 & 8666762_scaffold & 116899 & 117141 & 100 & 2 & 2 \\
\hline \multirow[t]{2}{*}{ DRB Exon 2} & 8670257_scaffold & $1687^{\mathrm{a}}$ & $1418^{\mathrm{a}}$ & 99.6 & 4 & N/A \\
\hline & 8666762_scaffold & $128830^{\mathrm{a}}$ & $128561^{a}$ & 99.6 & & N/A \\
\hline \multirow[t]{2}{*}{ DQA Exon 2} & 8670390_scaffold & 8345 & 8593 & 96.4 & 0 & 0 \\
\hline & 8669331_scaffold & $27189^{a}$ & $26941^{a}$ & 82.3 & & 0 \\
\hline DQB Exon $2^{b}$ & \multicolumn{4}{|c|}{ No significant matches detected } & 10 & N/A \\
\hline
\end{tabular}

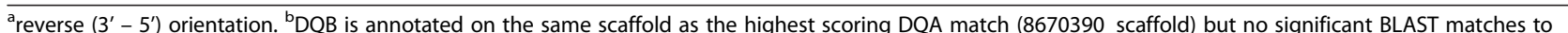
exon 2 were detected 





is consistent with observations from Sanger sequencing (Table 6, Figure $8 \mathrm{~b} / \mathrm{c}$ ). The partial $D Q A$ exon 2 sequence retrieved from the historical sample AQ40 was identical to the $D Q A$ exon 2 sequence found in modern dromedaries. Selected chromatograms are available in Additional file 4 .

\section{$D Q B$ exon 2}

Due to the location of the primers located within the exon 2, the sequence analyzed was truncated and contained 181 bp. A $12 \mathrm{bp}$ long insertion, as compared to the corresponding bovine sequence (266 bp) [GenBank: S43261.1|:53-319] (Fig. 9) was detected in all three species. Twenty-one polymorphic sites were observed within this sequence across all three species, resulting in altogether 16 non-synonymous substitutions as shown in Fig. 9. The C. ferus sequences revealed a total of 16 SNPs, resulting in 11 non-synonymous substitutions. Fifteen SNPs were observed in the $D Q B$ exon 2 of C. bactrianus. Among these 15 SNPs, 11 were non-






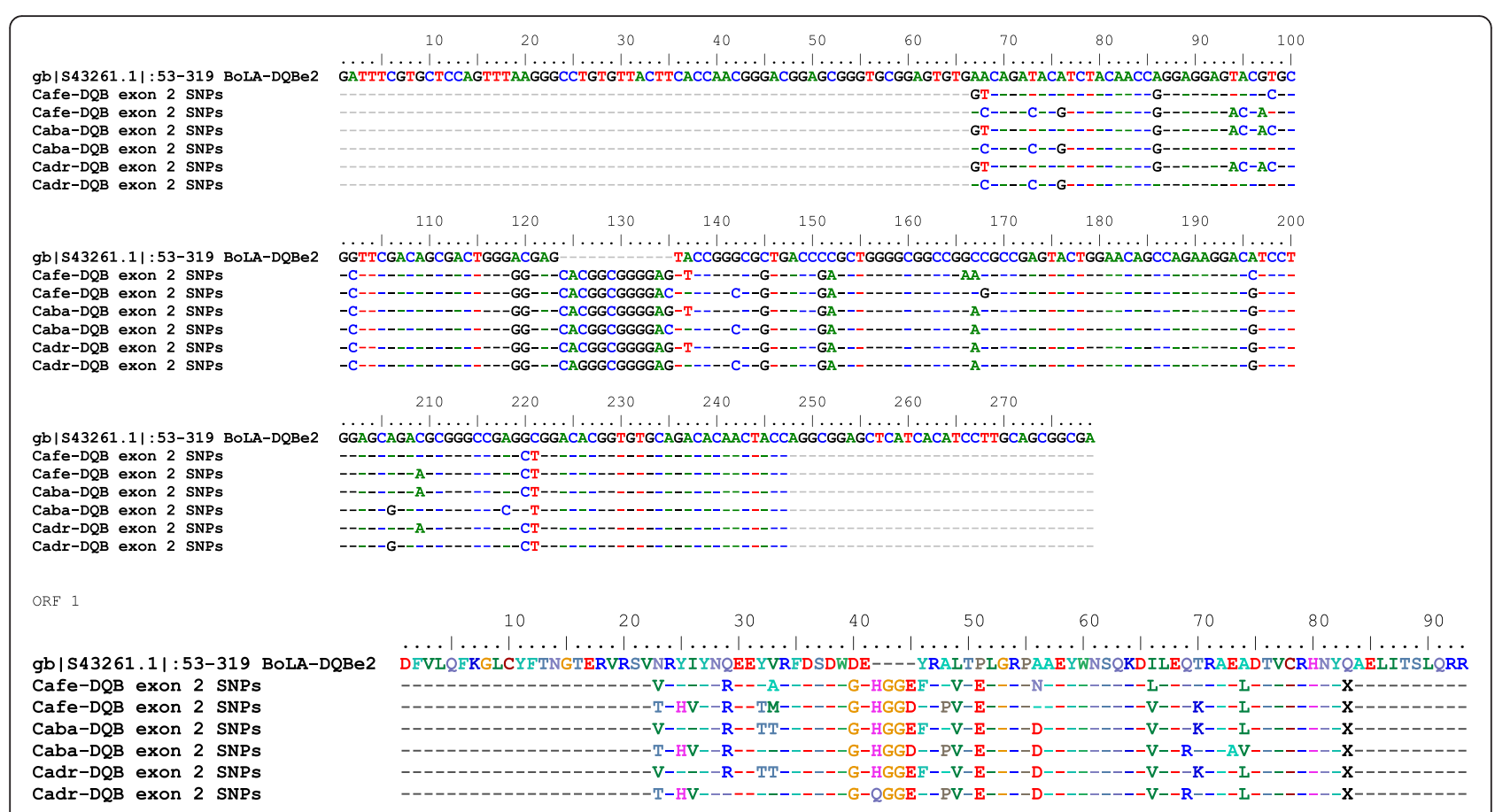

Fig. 9 SNPs identified in the partial sequence of DQB exon 2 of Camelus ferus (Cafe), Camelus bactrianus (Caba) and Camelus dromedarius (Cadr) and their in silico translation

synonymous substitutions. In C. dromedarius, 14 SNPs with 11 non-synonymous substitutions were found (Fig. 9). Selected chromatograms are available in Additional file 5.

\section{Discussion}

In this study we combined cytogenetics, Sanger sequencing, and NGS to investigate the MHC in Old World camels. Our results indicated that the MHC region of these three species is structurally similar to that of New World camelids (llamas) and of bovids. These results are in agreement with data previously reported for llamas $[32,57]$. A combination of different techniques was necessary to recapitulate the MHC organization. We have shown that even whole genome sequences with coverage $65 x$ could not provide complete information on the MHC region in camelids and confirmed that targeted re-sequencing is needed to resolve the complexity of this region. New techniques, like long-read NGS, are needed to further characterize this important genomic region.

FISH analysis located the MHC region on the short arm of chromosome 20 in dromedaries. Taking into consideration the high similarities between camelid genomes and karyotypes in general, we may assume that chromosomes Cba 20 and Cfe 20 carry the MHC in the other two species as well. The structure of the entire chromosome is evolutionary conserved between camelids and bovids and these chromosomes are homologous to the MHC-carrying llama and bovine chromosomes LPA20 and Bta 23, respectively [32, 55]. The MHC of camels is similar to the MHC of cattle and other bovids not only in terms of their physical location, but also of their genetic structure. The order and orientation of the three major regions, i.e. centromere - class II - class III and class I as inferred from the WGS [25] were directly visualized by the FISH (Fig. 1).

A general comparison between the three camel species showed substantial similarity and shared features among their MHCs. They seem to reflect similarities of their genomes in general rather than to be specific for the $\mathrm{MHC}$ region. Comparisons of mitochondrial genomes revealed 99 \% sequence identity between $C$. ferus and $C$. bactrianus, and $93 \%$ identity between C. bactrianus and C. dromedarius (Mohandesan et al. in prep). High number of shared alleles as well as the overall low allele count as shown in the Fig. 4 for respective loci suggests that the conservation of these sequences among camelids is still higher than similarities with other species and among these other species as shown in Table 7. Sequence IDs used for comparison in Table 7 are listed in Table 8. In camels, the DRA locus seems to be the most conserved, $D R B$ the most divergent, with $D Q A$ intermediate.

From the evolutionary perspective, only genes coding for antigen-presenting molecules, i.e. class I and class II, belong to the ancestral MHC [58]. Their genetic variation is functionally important and it was shown to be associated with a variety of diseases. Their complexity 
Table 7 Overall exon 2 similarities of nucleotide sequences (n) and peptide sequences ( $p$ ) between camel, cattle, human, pig, horse, sheep and goat. Due to high similarity between all camels only Camelus ferus sequences were used as reference Accession numbers are listed in Table 8

\begin{tabular}{|c|c|c|c|c|c|c|}
\hline & \multicolumn{2}{|l|}{ DRA } & \multicolumn{2}{|l|}{ DRB } & \multicolumn{2}{|l|}{ DQA } \\
\hline & (n) & (p) & (n) & (p) & (n) & (p) \\
\hline cattle & 0.894 & 0.84 & 0.785 & 0.652 & 0.835 & 0.768 \\
\hline human & 0.919 & 0.901 & 0.77 & 0.674 & 0.843 & 0.78 \\
\hline pig & 0.939 & 0.901 & 0.756 & 0.64 & 0.835 & 0.805 \\
\hline horse & 0.923 & 0.864 & 0.752 & 0.573 & 0.819 & 0.756 \\
\hline sheep & 0.77 & 0.852 & 0.732 & 0.607 & 0.827 & 0.768 \\
\hline goat & 0.774 & 0.864 & 0.766 & 0.629 & 0.835 & 0.78 \\
\hline
\end{tabular}

and genetic organization require specific methodological approaches, differing between the class I and class II regions. Here, we studied functionally important and usually the most polymorphic class II genes $D R A, D R B$, $D Q A$ and $D Q B$, encoding the $\alpha$ and $\beta$ chains composing functional $D R$ and $D Q$ antigen-presenting dimers [1].

MHC class II genes are highly variable between different species due to differences in numbers of genes evolved by duplications as well as in the extent of polymorphism between individual genes $[59,60]$. Intraspecific variation may be due not only to high numbers of genes and alleles, but also to variation in gene number among individual haplotypes observed in different species, including cattle [61]. It was therefore surprising to observe low variability in the MHC class II exon 2 genomic sequences in the three camelid species. Low numbers of alleles and high numbers of homozygotes in all loci, and many alleles shared across species substantially differ from most other mammalian species with tens or even hundreds of alleles and a high degree of heterozygosity [7]. Based on our observations, we extended the numbers of animals analyzed making sure that the camels studied originated from all major regions in the world (see Additional file 1). Three approaches (Sanger, NGS, cloning) were used to confirm the sequences retrieved and different primer pairs spanning overlapping regions were used to minimize allelic drop-outs. In addition, zoo-primers amplifying canonical
MHC class II loci in other species were used. All these approaches produced similar results. The extent of allelic variation identified in groups of animals comparable to studies of other mammalian models seems to be unusually low in this particular family. We only can speculate whether there is another methodological limitation, which avoided us to assess the entire range of existing variation or whether there are reasons related to the biology of this family. Our data did not provide information about the extent of genetic variation in other MHC genes, especially in the class I loci. Lower MHC variation in different species is usually associated with (i) bottlenecks or reduced sizes of specific populations [62], (ii) pathogen-poor environment [63], (iii) particular traits of social interactions such as monogamy combined with small population numbers and low reproduction rate [64], or (iv) limited chance for lateral pathogen transmission [65]. Out of these, the pathogen poor environment is an attractive hypothesis. We observed a lower overall genomic heterozygosity in dromedaries compared to domestic and wild Bactrian camels, which could hint to a generally lower genetic diversity in dromedaries (Fitak et al. in prep). However, for the MHC region, the low level of genetic diversity was observed in all three species.

The whole genome sequences available combined with the results of this study still did not allow us to determine the complete number of different class II loci. In most species, the DRA molecule is encoded by a single locus and it is usually monomorphic or with very little variation $[66,67]$. Our observation of two SNPs with one nonsynonymous substitution is in agreement with this general pattern. All three camel species shared identical alleles, although their distribution differed according to species. Interestingly, the allele $D R A * 03$ prevailing in C. ferus (frequency $=0.444)$ was rarely observed in both domesticated species, C. bactrianus (0.05) and C. dromedarius (0.067).

The DR $\beta$ chain is usually encoded by multiple highly polymorphic loci [67]. Hundreds of alleles and variation in the numbers of loci were observed in primates [6]. However, in some species like musk-ox and fallow deer, the $D R B$ genes were found to be monomorphic [60]. In the three camel species, this was the only locus where species-

Table 8 Accession numbers of MHC class II exon 2 sequences used for comparison in Table 7

\begin{tabular}{llll}
\hline & DRA & DRB & DQA \\
\hline camel & gb|AGVR01020883.1|:30918-31163 & gb|AGVR01020883.1|:19231-19500 & gb|AGVR01020882.1|:14962-15210 \\
cattle & gb|DQ821713.1|:48-293 & gi|21668455:285-554 & gi|116078064:75-316 \\
human & gi|301171411:192-437 & gi|262072349|gb|GU066757.1| & gb|L34082.1|HUMMHDQAAA:14-262 \\
pig & gb|EU722917.1|:103-348 & gb|GU263817.1|:372-641 & gi|166065110|gb|EU195146.1| \\
horse & gb|JN035629.1|:26-271 & gb|JN035622.1|:267-536 & gb|JQ254060.1|:84-332 \\
sheep & gi|220981783:85-327 & gb|AH001247.2|SEG_SHPMHDQB0:204-473 & gb|M33304.1|SHPMHDQAA:359-607 \\
goat & gi|2618610:2117-2362 & gi|2575824|dbj|AB008347.1| & gb|AY464656.1|:96-344 \\
\hline
\end{tabular}


specific SNPs were observed and it seems to be the least conserved out of the loci studied. This corresponds to high inter-species variability observed in other mammals [68]. The lowest numbers of $D R B$ alleles were found in $C$. ferus, which is probably due to the limited number of individuals sampled of this species, originating from a single population and to the fact that wild camels experienced a population reduction of $80 \%$ over the last 100 years [69].

In most mammals, the $D Q A$ region is typically multilocus and highly polymorphic [70]. In cattle, variation in the number of $D Q A$ genes among individual haplotypes were reported [71-73]. Our in silico search of the camelid genomes revealed two possible $D Q A$ loci. However, the lower scoring locus seems to be a non-functional copy, as no other MHC genes could be found within the same scaffold (data not shown). Although several SNPs allowing multiple haplotype combinations were observed in the camel exon $2 D Q A$ nucleotide sequences, only three allelic haplotypes were observed, two of them shared by all three camel species although with different frequencies (Table 5). The $D Q A$ sequences were also highly similar to human sequences $(0.843$ and 0.780 for nucleotide and peptide sequences, respectively), and in contrast to the $D R$ loci, they showed high similarities with their cattle and pig orthologues (Table 7).

The $D Q B$ genomic region seems to be very complex in camels like in many other mammalian species, with many repetitive sequences of different types [7]. For this reason probably, the camel whole genome sequences published so far do not contain this region. The fact that we were unable to amplify full-length exon 2 sequences by using several pairs of $D Q B$ zoo-primers (human, cattle, equine) and that even high-coverage whole genome sequencing and/or NGS did not allow resolving the organization of the $D Q B$ region, is in agreement with this assumption. In this situation, we had to use the zooprimers GH 28 and 29, shown previously to amplify exon $2 D Q B$ sequences from at least two loci of multiple mammalian species $[36,74]$. The partial $D Q B$ exon 2 sequences obtained here contained high numbers of SNPs, with many non-synonymous substitutions. However, they showed the same patterns like the $D Q A$ locus, with very few individual haplotypes. As the $D Q B$ sequences obtained here are truncated, some additional variation may exist in these putative loci. A unique feature of the $D Q B$ exon 2 sequences is the $12 \mathrm{bp}$ insertion not observed in other mammalian species. Based on in silico translation, the putative polypeptide may be fully functional.

The study of highly polymorphic immune genes in ancient human (Denisovan) DNA samples revealed that adaptive introgression of archaic alleles has significantly shaped the modern human immune system [75]. Analyses of MHC genes from ancient domestic animal specimens thus can help to elucidate the historical events occurring during the domestication process. Most ancient DNA studies in domestic animals relied on mtDNA due to general difficulties associated with the recovery of nuclear DNA (nuDNA) from archaeozoological materials. Despite their importance, there have been no studies of ancient immune genes and their role in domestication and adaptation in livestock to date. This dataset represents the first MHC sequences retrieved from ancient camel specimens from hot and arid environments, which are notoriously the most unfavorable for the survival of DNA [76]. Although fragmentary at this stage, the data showed that some DRA haplotypes have been maintained over time. The three extra $\mathrm{C} / \mathrm{T}$ substitutions observed in AQ30 still need to be validated as true polymorphisms in additional (historical) samples, rather than representing ancient DNA damage commonly observed as $\mathrm{C}$ to $\mathrm{T}$ (complementary $\mathrm{G}$ to $\mathrm{A}$ ) change resulting from post-mortem cytosine deamination [77].

Previously, Antczak [23] reported on the existence of polymorphic MHC class I and class II genes and on MHClinked microsatellite repeats located in the Bactrian camel genome. A whole genome cytogenetic map including data on the MHC region is available for New World camelids [57]. However, no specific sequences were available for a comparison for the purposes of this study.

\section{Conclusion}

In summary, this study provided the first evidence that camels possess an MHC comparable to other mammalian species in terms of its genomic localization, organization and sequence similarity. This is the first complex report on the order of the three MHC major sub-regions in Old World camels, based on physical mapping. We described ancient variation at the $D R A$ locus, monomorphic in most species. The extent of molecular diversity of MHC class II genes seems to be substantially lower in Old World camels than in other mammalian species. The major part of the diversity could reside in the complex $D Q B$ region, which was difficult to resolve and remained unannotated in the whole genome sequences.

\section{Availability of supporting data}

All alignments and resulting phylogenetic trees are available at the TreeBASE (http://treebase.org/treebase-web/ home.html) under DOI http://purl.org/phylo/treebase/ phylows/study/TB2:S18850.

All sequences were submitted to GenBank (http:// www.ncbi.nlm.nih.gov/genbank/) and are available under accession numbers KT936396-KT936421.

\section{Additional files}

Additional file 1: List of animals included in the study. (PDF $220 \mathrm{~kb}$ ) Additional file 2: Chromatograms of selected DRA alleles. (PDF $218 \mathrm{~kb}$ ) 
Additional file 3: Chromatograms of selected DRB alleles. Rest of the alleles was inferred. (PDF 332 kb)

Additional file 4: Chromatograms of selected DQA alleles. (PDF $156 \mathrm{~kb}$ ) Additional file 5: Chromatograms of selected DQB sequences. (PDF $77 \mathrm{~kb}$ )

\section{Competing interests}

The authors declare that they have no competing interests.

\section{Authors' contributions}

PM: Participated in study design, carried out the primer design for recent DNA, carried out the sequence alignment, participated in data analysis, participated in drafting the manuscript. ME: Participated in study design, carried out the ancient DNA study, was involved in data analysis, participated in drafting the manuscript. FRR: Carried out the bioinformatic analysis, participated in drafting the manuscript. MP: Performed the FISH analysis. KS: Performed the FISH analysis. BPA: Participated in study design, provided the sample set, participated in drafting the manuscript. HP: Conceived the study, participated in its design and coordination, participated in drafting the manuscript. All authors have read the manuscript and approved its release in the current form.

\section{Acknowledgments}

Projects: The work was supported by the Central European Institute of Technology (CEITEC) CZ.1.05/1.1.00/02.0068, by the project Aktion (68p7), and by the Austrian Science Foundations (FWF; P24706-B25 to PB); PB is recipient of an APART fellowship (11506) of the Austrian Academy of Sciences. The authors would like to thank Drs. Joris Peters (Institute of Palaeoanatomy, Domestication Research and the History of Veterinary Medicine, Ludwig-Maximilian University, Munich, Germany), Moneeb Qablan (University of Veterinary and Pharmaceutical Sciences Brno, Czech Republic), Elena Ciani and Ingrid Alloggio (University of Bari, Italy) for providing selected samples.

\section{Author details}

Department of Animal Genetics, Veterinary and Pharmaceutical University, Brno, Czech Republic. ${ }^{2}$ Ceitec VFU, RG Animal Immunogenomics, Brno, Czech Republic. ${ }^{3}$ Research Institute of Wildlife Ecology, Vetmeduni Vienna, Vienna, Austria. ${ }^{4}$ Institute of Population Genetics, Vetmeduni Vienna, Vienna, Austria. ${ }^{5}$ Department of Biology, Duke University, Durham, NC, USA. ${ }^{6}$ Department of Genetics and Reproduction, Veterinary Research Institute, Brno, Czech Republic.

\section{Received: 9 November 2015 Accepted: 18 February 2016}

\section{Published online: 01 March 2016}

\section{References}

1. Janeway CA, Travers P, Walport M, Shlomchik MJ. Immunobiology: The Immune System in Health and Disease. 5th ed. New York: Garland Science: 2001. http://www.ncbi.nlm.nih.gov/books/NBK27156/.

2. Meyer D, Thomson G. How selection shapes variation of the human major histocompatibility complex: a review. Ann Hum Genet. 2001;65:1-26.

3. Hedrick PW. Evolutionary genetics of the major histocompatibility complex. American Naturalist. 1994:143:945-964.

4. Reche PA, Reinherz EL. Sequence variability analysis of human class I and class II MHC molecules: functional and structural correlates of amino acid polymorphisms. J Mol Biol. 2003;331:623-41.

5. Cho S, Attaya M, Monaco JJ. New class II-like genes in the murine MHC. Nature. 1991;353:573-6.

6. Bontrop RE, Otting N, Groot NG, Doxiadis GG. Major histocompatibility complex class II polymorphisms in primates. Immunol Rev. 1999;167:339-50.

7. Robinson J, Halliwell JA, McWilliam H, Lopez R, Marsh SGE. IPD—the Immuno Polymorphism Database. Nucleic Acids Res. 2013;41:D1234-40.

8. He Y, Xi D, Leng J, Qian T, Jin D, Chen T, et al. Genetic variability of MHC class II DQB exon 2 alleles in yak (Bos grunniens). Mol Biol Rep. 2014;41: 2199-206.

9. Alcaide M, Munoz J, Martínez-de la Puente J, Soriguer R, Figuerola J. Extraordinary MHC class II B diversity in a non-passerine, wild bird: the Eurasian Coot Fulica atra (Aves: Rallidae). Ecol Evol. 2014;4:688-98.

10. Wegner KM, Reusch TBH, Kalbe M. Multiple parasites are driving major histocompatibility complex polymorphism in the wild. J Evol Biol. 2003;16: 224-32.
11. Bulliet RW. The Camel and the Wheel, vol. 56. Cambridge, MA: Harvard University Press; 1975. p. 340.

12. Burger PA. Genetic Traces of Domestication in Old World Camelids. In: Knoll EM, Burger PA, editors. Camels in Asia and North Africa: Interdisciplinary Perspectives on Their Past and Present Significance. Vienna: Austrian Academy of Science Press; 2012. p. 17-28.

13. Wu H, Guang X, Al-Fageeh MB, Cao J, Pan S, Zhou H, et al. Camelid genomes reveal evolution and adaptation to desert environments. Nat Commun. 2014:5:1-9.

14. Ji R, Cui P, Ding F, Geng J, Gao H, Zhang H, et al. Monophyletic origin of domestic bactrian camel (Camelus bactrianus) and its evolutionary relationship with the extant wild camel (Camelus bactrianus ferus). Anim Genet. 2009:40:377-82.

15. Silbermayr K, Orozco-terWengel P, Charruau P, Enkhbileg D, Walzer C, Vog $C_{\text {, et }}$ al. High mitochondrial differentiation levels between wild and domestic Bactrian camels: a basis for rapid detection of maternal hybridization. Anim Genet. 2010:41:315-8.

16. Jirimutu, Wang $Z$, Ding $G$, Chen $G$, Sun $Y$, Sun $Z$, Zhang $H$, Wang $L$, Hasi S, Zhang Y, Li J, Shi Y, Xu Z, He C, Yu S, Li S, Zhang W, Batmunkh M, Ts B, Narenbatu, Unierhu, Bat-Ireedui S, Gao H, Baysgalan B, Li Q, Jia Z, Turigenbayila, Subudenggerile, Narenmanduhu, Wang Z, et al. Genome sequences of wild and domestic bactrian camels. Nat Commun. 2012; 3:1202

17. Wernery $U$, Kaaden $O R$. Foot-and-mouth disease in camelids: a review. Vet J. 2004;168:134-42.

18. Dirie MF, Abdurahman O. Observations on little known diseases of camels (Camelus dromedarius) in the Horn of Africa. Revue scientifique et technique-Office international des épizooties. 2003:22:1043-50.

19. Azhar El, El-Kafrawy SA, Farraj SA, Hassan AM, Al-Saeed MS, Hashem AM, et al. Evidence for Camel-to-Human Transmission of MERS Coronavirus. N Engl J Med. 2014;370(26):2499-505

20. Hemida MG, Elmoslemany A, Al-Hizab F, Alnaeem A, Almathen F, Faye B, et al. Dromedary Camels and the Transmission of Middle East Respiratory Syndrome Coronavirus (MERS-CoV). Transbound Emerg Dis. 2015. doi:10. 1111/tbed.12401.

21. Muyldermans S, Baral TN, Retamozzo VC, De Baetselier P, De Genst E, Kinne J, et al. Camelid immunoglobulins and nanobody technology. Vet Immunol Immunopathol. 2009;128:178-83.

22. Tillib SV, Vyatchanin AS, Muyldermans S. Molecular analysis of heavy chain-only antibodies of Camelus bactrianus. Biochem Mosc. 2014;79:1382-90.

23. Antczak D. Major Histocompatibility Complex Genes Of The Dromedary Camel. In: Qatar Foundation Annual Research Conference. Doha, Qatar: BIOP 015:2013.

24. Burger PA, Palmieri N. Estimating the Population Mutation Rate from a de novo Assembled Bactrian Camel Genome and Cross-Species Comparison with Dromedary ESTs. J Hered. 2013;105(6):839-46.

25. Fitak RR, Mohandesan E, Corander J, Burger PA. The de novo genome assembly and annotation of a female domestic dromedary of North African origin. Mol Ecol Resour. 2015. doi:10.1111/1755-0998.12443.

26. Green ED, Guyer MS, Institute NHGR. Charting a course for genomic medicine from base pairs to bedside. Nature. 2011:470:204-13.

27. Lighten J, van Oosterhout C, Bentzen P. Critical review of NGS analyses for de novo genotyping multigene families. Mol Ecol. 2014;23:3957-72.

28. Green MR, Sambrook J. Molecular Cloning: A Laboratory Manual, vol. 1. New York: Cold Spring Harbor Laboratory Press; 2012.

29. Knapp M, Clarke AC, Horsburgh KA, Matisoo-Smith EA. Setting the stage-building and working in an ancient DNA laboratory. Annals of Anatomy-Anatomischer Anzeiger. 2012;194:3-6.

30. Rohland N, Hofreiter M. Comparison and optimization of ancient DNA extraction. Biotechniques. 2007:42:343.

31. Rohland N, Siedel H, Hofreiter M. A rapid column-based ancient DNA extraction method for increased sample throughput. Mol Ecol Resour. 2010; 10:677-83.

32. Avila F, Das PJ, Kutzler M, Owens E, Perelman P, Rubes J, et al. Development and application of camelid molecular cytogenetic tools. J Hered. 2014; 201(105):858-69.

33. Basic Local Alignment Search Tool. http://blast.ncbi.nlm.nih.gov/Blast.cgi. Accessed 15 Oct 2015

34. Rozen S, Skaletsky H. Primer3 on the WWW for general users and for biologist programmers. In: Bioinformatics methods and protocols. New York, NY. USA: Springer; 1999. 365-386. 
35. Sigurdardóttir S, Borsch C, Gustafsson K, Andersson L. Gene duplications and sequence polymorphism of bovine class II DQB genes. Immunogenetics. 1992;35:205-13.

36. Gyllensten UB, Lashkari D, Erlich HA. Allelic diversification at the class II DQB locus of the mammalian major histocompatibility complex. Proc Natl Acad Sci. 1990;87:1835-9.

37. Sff_extract. http://bioinf.comav.upv.es/sff_extract/index.html. Accessed 9 July 2014.

38. GS de novo Assembler. http://www.454.com/products/analysis-software/. Accessed 9 July 2014

39. FastQC. http://www.bioinformatics.babraham.ac.uk/projects/fastqc/. Accessed 10 July 2014.

40. Shrestha R, Lubinsky B, Bansode V, Moinz M, McCormack G, Travers S. QTrim: a novel tool for the quality trimming of sequence reads generated using the Roche/454 sequencing platform. BMC Bioinformatics. 2014;15:33.

41. Martin M. Cutadapt removes adapter sequences from high-throughput sequencing reads. EMBnet J. 2011;17:10-2.

42. Li H. Aligning sequence reads, clone sequences and assembly contigs with BWA-MEM. arXiv preprint arXiv:13033997 2013.

43. Li H, Handsaker B, Wysoker A, Fennell T, Ruan J, Homer N, et al. 1000 Genome Project Data Processing Subgroup. The Sequence Alignment/Map format and SAMtools. Bioinformatics. 2009;25:2078-9.

44. Robinson JT, Thorvaldsdóttir H, Winckler W, Guttman M, Lander ES, Getz G, et al. Integrative genomics viewer. Nat Biotechnol. 2011;29:24-6.

45. McKenna A, Hanna M, Banks E, Sivachenko A, Cibulskis K, Kernytsky A, et al. The Genome Analysis Toolkit: a MapReduce framework for analyzing next-generation DNA sequencing data. Genome Res. 2010;20:1297-303.

46. DePristo MA, Banks E, Poplin R, Garimella KV, Maguire JR, Hartl C, et al. A framework for variation discovery and genotyping using next-generation DNA sequencing data. Nat Genet. 2011;43:491-8.

47. Hall TA. BioEdit: a user-friendly biological sequence alignment editor and analysis program for Windows 95/98/NT. In: Nucleic acids symposium series, vol. 41. 1999. p. $95-8$

48. Auwera GA, Carneiro MO, Hartl C, Poplin R, del Angel G, Levy-Moonshine A, et al. From FastQ data to high-confidence variant calls: the genome analysis toolkit best practices pipeline. Curr Protoc Bioinformatics. 2013:11(1110):11.10.1-11.10.33.

49. Jukes TH, Cantor CR. Evolution of protein molecules. Mamm Protein Metab. 1969;3:21-132.

50. Kimura M. A simple method for estimating evolutionary rates of base substitutions through comparative studies of nucleotide sequences. J Mol Evol. 1980;16:111-20

51. Akaike H. A new look at the statistical model identification. Automatic Control, IEEE Transactions on. 1974;19:716-23.

52. Saitou N, Nei M. The neighbor-joining method: a new method for reconstructing phylogenetic trees. Mol Biol Evol. 1987;4:406-25.

53. Tamura K, Stecher G, Peterson D, Filipski A, Kumar S. MEGA6: molecular evolutionary genetics analysis version 6.0. Mol Biol Evol. 2013;30:2725-9.

54. Klein J, Bontrop RE, Dawkins RL, Erlich HA, Gyllensten UB, Heise ER, Jones PP, Parham P, Wakeland EK, Watkins DI. Nomenclature for the major histocompatibility complexes of different species: a proposal. In: Solheim BG, Ferrone S, Möller E. editors. The HLA System in Clinical Transplantation. Berlin, Heidelberg, Germany: Springer. 1993; 407-411.

55. Balmus G, Trifonov VA, Biltueva LS, O'Brien PC, Alkalaeva ES, Fu B, et al. Cross-species chromosome painting among camel, cattle, pig and human: further insights into the putative Cetartiodactyla ancestral karyotype. Chromosom Res. 2007;15:499-514.

56. Kumánovics A, Takada T, Lindahl KF. Genomic organization of the mammalian MHC. Annu Rev Immunol. 2003:21:629-57.

57. Avila F, Baily MP, Perelman P, Das PJ, Pontius J, Chowdhary R, et al. A Comprehensive Whole-Genome Integrated Cytogenetic Map for the Alpaca (Lama pacos). Cytogenet Genome Res. 2014;144:196-207.

58. Klein J, Figueroa F. Evolution of the major histocompatibility complex. Crit Rev Immunol. 1985;6:295-386.

59. Parham P. Virtual reality in the MHC. Immunol Rev. 1999;167:5-15.

60. Mikko S, Røed K, Schmutz S, Andersson L. Monomorphism and polymorphism at Mhc DRB loci in domestic and wild ruminants. Immunol Rev. 1999;167:169-78.

61. Andersson L, Rask L. Characterization of the MHC class II region in cattle. The number of DQ genes varies between haplotypes. Immunogenetics. $1988 ; 27: 110-20$
62. Bollmer JL, Vargas FH, Parker PG. Low MHC variation in the endangered Galapagos penguin (Spheniscus mendiculus). Immunogenetics. 2007;59: 593-602.

63. Weber DS, Van Coeverden De Groot PJ, Peacock E, Schrenzel MD, Perez DA, Thomas S, et al. Low MHC variation in the polar bear: implications in the face of Arctic warming? Anim Conserv. 2013;16:671-83.

64. Sommer S, Tichy $\mathrm{H}$. Major histocompatibility complex (MHC) class ॥ polymorphism and paternity in the monogamous Hypogeomys antimena, the endangered, largest endemic Malagasy rodent. Mol Ecol. 1999;8:1259-72.

65. Ellegren H, Mikk S, Wallin K, Andersson L. Limited polymorphism at major histocompatibility complex (MHC) loci in the Swedish moose A. alces. Mol Ecol. 1996;5:3-9.

66. Zhou H, Hickford JGH, Fang Q, Byun SO. Short Communication: Identification of Allelic Variation at the Bovine DRA Locus by Polymerase Chain Reaction-Single Strand Conformational Polymorphism. J Dairy Sci. 2007;90:1943-6.

67. Takada T, Kikkawa Y, Yonekawa H, Amano T. Analysis of goat MHC class II DRA and DRB genes: identification of the expressed gene and new DRB alleles. Immunogenetics. 1998;48:408-12.

68. Takeshima S, Saitou N, Morita M, Inoko H, Aida Y. The diversity of bovine MHC class II DRB3 genes in Japanese Black, Japanese Shorthorn, Jersey and Holstein cattle in Japan. Gene. 2003;316:111-8.

69. Yadamsuren, A, Dulamtseren E, Reading RP. The conservation status and management of wild camels in Mongolia. In: Knoll EM, Burgr PA, editors. Camels in Asia and North Africa. Interdisciplinary perspectives on their significance in past and present.Vienna. Austrian Academy of Sciences Press. 2012; p 45-54.

70. Ballingall KT, Steele P, Lantier I, Cotelli M, Todd H, Lopez G, et al. An ancient interlocus recombination increases class II MHC DQA diversity in sheep and other Bovidae. Anim Genet. 2015:46:333-6.

71. Ballingall KT, Luyai A, McKeever DJ. Analysis of genetic diversity at the DQA loci in African cattle: evidence for a BoLA-DQA3 locus. Immunogenetics. 1997;46:237-44.

72. Moutou K, Koutsogiannouli E, Stamatis C, Billinis C, Kalbe C, Scandura $\mathrm{M}$, et al. Domestication does not narrow MHC diversity in Sus scrofa. Immunogenetics. 2013;65:195-209.

73. Behl JD, Verma NK, Tyagi N, Mishra P, Behl R, Joshi BK. The Major Histocompatibility Complex in Bovines: A Review. ISRN Vet Sci. 2012;2012:1-12.

74. Hořin P, Matiašovic J. A second locus and new alleles in the major histocompatibility complex class II (ELA-DQB) region in the horse. Anim Genet. 2002:33:196-200.

75. Abi-Rached L, Jobin MJ, Kulkarni S, McWhinnie A, Dalva K, Gragert L, et al. The shaping of modern human immune systems by multiregional admixture with archaic humans. Science. 2011;334:89-94.

76. Hofreiter M, Paijmans JL, Goodchild H, Speller CF, Barlow A, Fortes GG, et al. The future of ancient DNA: Technical advances and conceptual shifts. BioEssays. 2015:37:284-93.

77. Briggs AW, Stenzel U, Meyer M, Krause J, Kircher M, Pääbo S. Removal of deaminated cytosines and detection of in vivo methylation in ancient DNA. Nucleic Acids Res. 2010:38:87.

\section{Submit your next manuscript to BioMed Central and we will help you at every step:}

- We accept pre-submission inquiries

- Our selector tool helps you to find the most relevant journal

- We provide round the clock customer support

- Convenient online submission

- Thorough peer review

- Inclusion in PubMed and all major indexing services

- Maximum visibility for your research

Submit your manuscript at www.biomedcentral.com/submit 